\title{
Monitoramento e estimativa da produtividade de soja auxiliados pelo modelo regional de previsão de tempo ETA \\ (doi:10.4136/ambi-agua.10)
}

\author{
Rodrigo Rizzi'; Bernardo Friedrich Theodor Rudorff²; Ramon Morais de Freitas²; \\ Denise Cybis Fontana ${ }^{3}$
}

\author{
1Universidade Federal de Pelotas (UFPel) \\ E-mail: rodrigo.rizzi@ufpel.edu.br \\ 2Instituto Nacional de Pesquisas Espaciais (INPE) \\ E-mail \{bernardo, ramon\}@dsr.inpe.br \\ ${ }^{3}$ Universidade Federal do Rio Grande do Sul (UFRGS) \\ E-mail dfontana@ufrgs.br
}

\section{RESUMO}

O objetivo deste trabalho foi monitorar e estimar a produtividade de soja por meio de um modelo agronômico em um Sistema de Informações Geográficas (SIG), utilizando os dados meteorológicos gerados pelo modelo regional de previsão de tempo Eta. O modelo agronômico permitiu a produção de mapas quinzenais de índices de penalização e estimativa da produtividade da soja para os estados do Mato Grosso do Sul, Paraná e São Paulo, safra 2004/05. O resultado mostrou que os dados meteorológicos do modelo Eta, incorporados em um modelo agronômico, permitem monitorar, no tempo e no espaço, a cultura da soja. Além disso, os dados meteorológicos do Eta são disponibilizados em tempo quase real, permitindo uma rápida avaliação, objetiva e quantitativa, do efeito das condições meteorológicas sobre a produção da soja.

Palavras-chave: estatísticas agrícolas; modelagem; dados meteorológicos; índice de penalização.

\section{Soybean grain yield monitoring and estimation aided by the mesoscale ETA Weather Forecast model}

\begin{abstract}
The objective of this work was to monitor and estimate soybean grain yield based on an agronomical model coupled to a Geographic Information System (GIS) using meteorological data generated by the Eta mesoscale weather forecast model. The agronomic model produced bi-weekly penalizing indices maps and soybean grain yield estimates for the States of Mato Grosso do Sul, Paraná and São Paulo, Brazil, during crop year 2004/05. Result showed that meteorological data from the Eta model, incorporated in an agronomic model, allowed monitoring, in time and space, the soybean crop. In addition, the meteorological data from Eta model are provided in almost real time allowing a quantitative and quick evaluation of weather conditions for soybean production.
\end{abstract}

Keywords: agricultural statistics; modeling; meteorological data; penalizing index. 
RIZZI, R; RUDORFF, B. F. T.; FREITAS, R. M.; FONTANA, D. C. Monitoramento e estimativa da produtividade de soja auxiliados pelo modelo regional de previsão de tempo ETA. Ambi-Agua, Taubaté, v. 1, n. 2, p. 28-38, 2006. (doi:10.4136/ambi-agua.10)

\section{INTRODUÇÃO}

No Brasil, o acompanhamento da safra agrícola das grandes culturas e o prognóstico da produção é realizado, predominantemente, com base na opinião de diversos profissionais ligados ao setor do agronegócio. A produção é dada pelo produto da área plantada com a produtividade, sendo que esta depende, principalmente, das condições meteorológicas que ocorrem ao longo da safra. O efeito da variabilidade do tempo e do clima sobre a produtividade é difícil de ser mensurado e, por isso, ainda é feito de forma subjetiva, dando margem à especulação. O recente avanço, tanto na disponibilidade de dados meteorológicos, quanto nos métodos de modelagem agronômica, pode conferir um caráter objetivo ao monitoramento da safra e à estimativa da produtividade.

A quantificação do efeito das variáveis agrometeorológicas sobre o crescimento e desenvolvimento das culturas pode ser realizada por meio de modelos agronômicos (Hoogenboom, 2000). Esses modelos podem ser incorporados aos Sistemas de Informações Geográficas (SIGs) para viabilizar o monitoramento das culturas não apenas no tempo, mas também no espaço (Hartkamp et al., 1999; Dang et al., 2000). Tal abordagem aponta os períodos dentro do ciclo das culturas e as regiões cultivadas em que o impacto das condições meteorológicas adversas sobre a produtividade foi mais expressivo (Berka et al., 2003; Rizzi, 2004).

As informações coletadas pelas estações meteorológicas são a principal fonte de dados para esses modelos. Entretanto, essas estações têm uma distribuição esparsa, em especial na zona rural, dificultando a utilização desses dados para muitas regiões de cultivo intenso, particularmente na fronteira agrícola. Além disso, os dados coletados por uma estação representam apenas uma pequena região em torno da qual e, muitas vezes, são adquiridos ou armazenados numa escala temporal inadequada para os modelos (Hoogenboon, 2000). Aliada a essas restrições está a dificuldade de acesso a esses dados em tempo oportuno, podendo inclusive inviabilizar o monitoramento da safra agrícola de modo a possibilitar a tomada de decisões em tempo hábil.

Uma alternativa aos dados coletados pelas estações são aqueles provenientes de modelos numéricos de previsão de tempo, os quais podem ser disponibilizados diariamente e para grandes regiões, imediatamente após a sua geração. O modelo regional de previsão de tempo Eta (letra grega $\eta$ ) foi desenvolvido pela Universidade de Belgrado em conjunto com o Instituto de Hidrometeorologia da Iugoslávia (Mesinger et al., 1988). Esse modelo foi instalado no Centro de Previsão de Tempo e Estudos Climáticos (CPTEC) do Instituto Nacional de Pesquisas Espaciais (INPE), no final de 1996, para complementar a previsão numérica de tempo que vem sendo realizada desde o início de 1995, a partir do modelo de circulação geral atmosférica (Chan, 1996). O CPTEC disponibiliza, diariamente, previsões geradas pelo modelo Eta para diversos elementos meteorológicos. Os dados possuem resolução espacial horizontal em versões de 40 x $40 \mathrm{~km}$ e 20 x $20 \mathrm{~km}$, esta com caráter operacional desde o final de 2005, e cobrem a maior parte da América do Sul.

Este trabalho tem por objetivo monitorar e estimar a produtividade de soja por meio de um modelo agronômico em um SIG, utilizando dados meteorológicos gerados pelo modelo regional de previsão de tempo Eta.

\section{MATERIAL E MÉTODOS}

Como área de estudo foi selecionada uma região de cultivo intenso de soja, que abrange parte dos estados do Paraná, São Paulo e Mato Grosso do Sul (Figura 1). A safra da 
soja analisada foi a de 2004/05, muito prejudicada por condições meteorológicas adversas, sobretudo a partir do mês de fevereiro desse período.

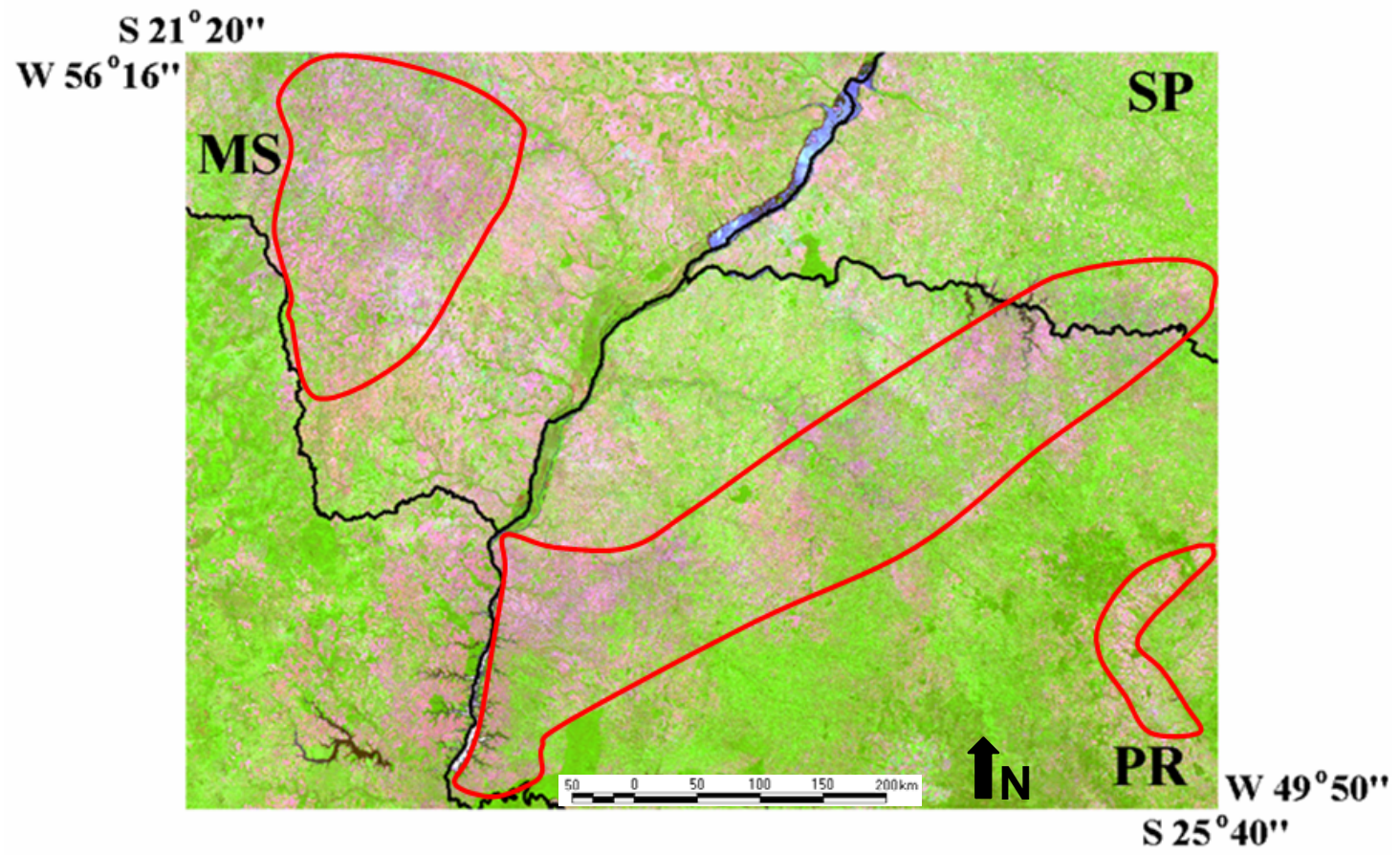

Figura 1. Área de estudo com a respectiva localização geodésica e o limite político estadual (linha preta), indicando as regiões de maior concentração de cultivo de soja (linha vermelha) sobre uma imagem do sensor MODIS.

O monitoramento da safra e a estimativa da produtividade da soja foram realizados por meio de um modelo agronômico, denominado AGRO (Berka et al, 2003), integrado ao SIG do aplicativo SPRING (Sistema de PRocessamento de INformações Georreferenciadas; www.dpi.inpe.br/spring) que reúne funções de processamento de imagens, análise espacial, modelagem numérica do terreno e consulta a bancos de dados espaciais (Câmara et al., 1996).

O modelo AGRO tem por base o Relatório $\mathrm{N}^{0} 33$ da FAO (Food and Agriculture Organization) de Doorenbos e Kassam (1979). Rudorff e Batista (1990) utilizaram a metodologia descrita por esses autores e desenvolveram um modelo com programação em linguagem FORTRAN para estimar a produtividade em locais específicos. Berka et al. (2003) empregaram as facilidades computacionais da análise espacial e dos SIGs para espacializar o modelo a fim de obter a estimativa da produtividade para grandes regiões. Outro avanço recente nesse modelo foi a introdução dos valores do índice de área foliar (IAF) estimados por meio das imagens do sensor MODIS a bordo do satélite Terra (Rizzi, 2004).

As variáveis meteorológicas necessárias ao modelo AGRO são: temperatura média do ar

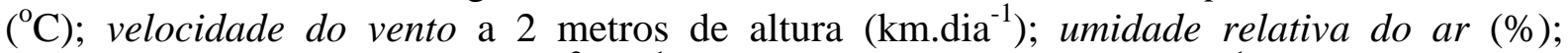
radiação solar incidente (cal. $\left.\mathrm{cm}^{-2} \cdot \mathrm{dia}^{-1}\right)$ e precipitação pluvial $\left(\mathrm{mm} \cdot \mathrm{dia}^{-1}\right)$. São necessários, ainda, dados referentes à altitude e à capacidade de armazenamento de água disponível (CAD) dos solos. Para o dado de altitude, foi utilizado um modelo digital de elevação com resolução espacial de aproximadamente $1 \times 1 \mathrm{~km}$ (GTOPO), disponibilizado pelo Eros Data Center-USGS (www.usgs.gov). Para o dado referente à CAD, foi utilizado um mapa de classificação de solos em escala 1:750.000 (Brasil, 1973). Os valores de CAD (50, 70 ou 100mm) foram atribuídos para cada classe pedológica, de acordo com Embrapa (1999) e Farias et al. (2000). 
As previsões do modelo regional Eta, operacional no CPTEC, são fornecidas duas vezes ao dia, com condição inicial às 00:00 e às 12:00 horas UTC e englobam previsões para sete dias em um intervalo de seis horas. No presente trabalho, os dados utilizados foram aqueles oriundos das previsões com condição inicial às 00:00 horas UTC e com resolução espacial horizontal de 40 x $40 \mathrm{~km}$, pois a versão que utiliza a grade de 20 x $20 \mathrm{~km}$ ainda não estava operacional por ocasião da aquisição dos dados. Como tecnicamente os dados referentes aos dois primeiros horários (00:00 e 06:00 horas UTC) estão associados a incertezas, principalmente para as variáveis velocidade do vento e precipitação pluvial, optou-se por utilizar os dados a partir da previsão das 12:00 horas UTC. Por exemplo, as variáveis meteorológicas do dia 01-11-05 dizem respeito às previsões das 12:00 e 18:00 horas UTC do dia 01-11-05 e das 00:00 e 06:00 horas UTC do dia 02-11-05 (condição inicial às 00:00 UTC de 01-11-05), totalizando 24 horas. Os dados das variáveis meteorológicas do modelo Eta foram transformados, primeiramente, para a escala diária, de modo a se assemelharem aos dados coletados pelas estações meteorológicas.

Para a variável precipitação pluvial, realizou-se o somatório das previsões dos quatro horários do dia, já que cada previsão representa a precipitação pluvial acumulada das últimas seis horas, de acordo com a Equação [1].

$$
P t=\sum_{t 1}^{t n} f_{i}
$$

em que $P t$ é a precipitação total (mm.dia ${ }^{-1}$ ) e $f$ é a precipitação pluvial acumulada (mm) observada nos horários 00:00, 06:00, 12:00 e 18:00 horas UTC.

Para a variável radiação solar incidente, fez-se o somatório das previsões para os quatro horários, visto que cada previsão representa a média das últimas seis horas, de acordo com a Equação [2].

$$
R S=c \cdot \sum_{t 1}^{t n} \bar{r}_{i}
$$

em que $R S$ é a radiação solar incidente acumulada (cal. $\mathrm{cm}^{-2}$. dia ${ }^{-1}$ ) nas últimas 24 horas; $\bar{r}$ é a radiação média observada $\left(\mathrm{W} . \mathrm{m}^{-2}\right)$ nos horários 00:00, 06:00, 12:00 e 18:00 horas UTC e $c$ é o fator de conversão da radiação solar incidente de W.m ${ }^{-2}$ para cal.cm ${ }^{-2} \cdot \mathrm{dia}^{-1}=0,51624$, de acordo com Allen et al. (1998).

Para a variável temperatura média do ar, utilizou-se a média entre a temperatura observada às 06:00 horas UTC (considerada neste trabalho como a temperatura mínima diária) e às 18:00 horas UTC (considerada neste trabalho como a temperatura máxima diária) (Allen et al., 1998), de acordo com a Equação [3].

$$
\operatorname{Tm}=\left(\frac{T_{06: 00}+T_{18: 00}}{2}\right)-x
$$

em que $T m$ é a temperatura média do ar $\left({ }^{\circ} \mathrm{C}\right)$; $T$ é a temperatura do ar $(\mathrm{K})$ observada às 06:00 e 18:00 horas UTC e $x$ é o fator de conversão da temperatura do ar de $\mathrm{K}$ para ${ }^{\circ} \mathrm{C}=$ 273,15 .

A variável velocidade do vento (magnitude), foi estimada a partir do vetor resultante das componentes cartesianas de vento U e V, de acordo com a Equação [4].

$$
V v=\sqrt{U^{2}+V^{2}}
$$

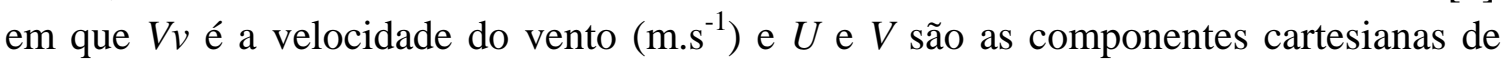
vento $\left(\mathrm{m} \cdot \mathrm{s}^{-1}\right)$. 
A média da velocidade do vento diária foi calculada a partir da Equação [5].

$V v m=\left(\frac{V v_{12: 00}+V v_{18: 00}+V v_{00: 00}}{3}\right) h . t$

em que $V v m$ é a média da velocidade do vento $\left(\mathrm{km} \cdot \mathrm{dia}^{-1}\right)$ para os horários 12:00, 18:00 e 00:00 horas UTC; $h$ é o fator de conversão de $\left(\mathrm{m}^{-1} \mathrm{~s}^{-1}\right)$ para $\left(\mathrm{km} \cdot \mathrm{dia}^{-1}\right)=86,4$ e $t$ é o fator de conversão da velocidade do vento de 10 para $2 \mathrm{~m}$ de altura $=0,75$, segundo Allen et al. (1998).

Como a variável umidade relativa do ar não é fornecida diretamente pelo modelo Eta, ela foi estimada a partir das variáveis temperatura de ponto de orvalho e temperatura do ar, a partir da equação de Clausius-Clapeyron, segundo Bolton (1980), apresentada na Equação [6].

$$
U R=100 \cdot \frac{6,112^{\frac{17,67 T d}{T d+243,5}}}{6,112^{\frac{17,67 T}{T+243,5}}}
$$

em que $U R$ é a umidade relativa (\%); Td é a temperatura de ponto de orvalho $\left({ }^{\circ} \mathrm{C}\right)$ e $T$ é a temperatura do ar $\left({ }^{\circ} \mathrm{C}\right)$. A média da umidade relativa do ar diária foi calculada a partir da Equação [7].

$$
U R m=\frac{\left(U R \%_{12: 00}\right)+\left(U R \%_{18: 00}\right)+\left(U R \%_{00: 00}\right)}{3}
$$

em que $U R m$ é a média da umidade relativa do ar (\%) e UR é a umidade relativa do ar (\%) para os horários 12:00, 18:00 e 00:00 horas UTC.

A escala temporal utilizada no modelo AGRO foi quinzenal, ou seja, são calculadas produtividades parciais a cada 15 dias, desde a implantação da cultura até a sua senescência, totalizando nove quinzenas (135 dias). O período de monitoramento das condições meteorológicas foi desde 01-11-04 (semeadura) até 15-03-05 (colheita), sendo definido de acordo com o calendário agrícola da soja na região em estudo. A produtividade final é resultado do somatório das produtividades parciais. Assim sendo, os dados meteorológicos diários foram organizados em médias quinzenais, salvo os dados referentes à precipitação pluvial, que foram somados quinzenalmente. Toda a metodologia que envolve a transformação dos dados do Eta para as escalas diária e quinzenal foi desenvolvida em uma rotina computacional de modo a minimizar o tempo entre a disponibilização dos dados e a sua utilização no modelo AGRO.

A resolução espacial empregada no modelo AGRO foi de $1 \times 1 \mathrm{~km}$. Dessa forma, todas as variáveis são representadas por meio de uma grade regular (matriz) em que cada ponto (pixel) corresponde a uma área de 1 x $1 \mathrm{~km}$ no terreno, sendo que o modelo calcula a produtividade da soja para cada ponto. Como os dados gerados pelo modelo Eta na versão utilizada são representados por uma grade regular de $40 \times 40 \mathrm{~km}$, eles foram espacializados ${ }^{1}$ pelo algoritmo média ponderada por quadrante, disponível no SIG do aplicativo SPRING, para a resolução espacial de 1 x $1 \mathrm{~km}$, de modo a compatibilizá-los à resolução espacial empregada no modelo AGRO.

A produtividade agrícola estimada pelo modelo AGRO utiliza a Equação [8] (Doorenbos; Kassam, 1979).

$$
P=P M\left[1-k y\left(1-\frac{E T r}{E T m}\right)\right]
$$

\footnotetext{
${ }^{1}$ Na espacialização, uma grade regular é gerada a partir de amostras pontuais por meio de uma interpolação espacial.
} 
em que $P$ é a produtividade real estimada pelo modelo (kg.ha $\left.{ }^{-1}\right), P M$ é a produtividade máxima $\left(\mathrm{kg}_{\mathrm{g}} \mathrm{ha}^{-1}\right)$, ky é o fator de resposta à produtividade que relaciona o déficit da evapotranspiração relativa (ETr/ETm) com a diminuição da produtividade e varia de acordo com o estádio em que a cultura se encontra (Doorenbos; Kassam, 1979). A média da produtividade da soja, em nível estadual, foi realizada por meio de um mapa contendo a divisão política estadual. Ressalta-se que somente os pixels representados por áreas de soja foram computados no cálculo da média estadual. Tais áreas foram definidas por meio de uma classificação multitemporal de imagens do sensor MODIS.

O acompanhamento do impacto das variáveis meteorológicas ao longo da estação de crescimento e desenvolvimento da soja foi realizado pelo modelo AGRO por meio do Índice de Penalização (IP) quinzenal, determinado pela Equação [9].

$$
I P=\frac{P a}{P m a}
$$

em que IP é o Índice de Penalização, $P a$ é a produtividade real estimada acumulada $\left(\mathrm{kg} \cdot \mathrm{ha}^{-1}\right)$ até a quinzena considerada e Pma é a produtividade máxima acumulada $\left(\mathrm{kg} . \mathrm{ha}^{-1}\right)$ até a quinzena considerada.

Dessa forma, o valor do IP expressa a produtividade real estimada obtida pela cultura em relação à sua produtividade potencial máxima, desde o início do seu ciclo até a quinzena considerada. Por exemplo, o IP 1,0 indica a melhor situação, quando as condições meteorológicas foram favoráveis e a cultura expressou 100\% do seu potencial produtivo, não havendo prejuízo para sua produtividade em função da disponibilidade meteorológica no período considerado.

\section{RESULTADOS E DISCUSSÃO}

A Figura 2 mostra os IPs quinzenais acumulados para a cultura da soja na região compreendida pelo estudo, safra 2004/05. Pela análise dos mapas de IP, na Figura 2, é perceptível a ocorrência de condições meteorológicas adversas durante o ciclo da soja em toda a região considerada, principalmente a partir de fevereiro, que se estendeu até o final do ciclo da cultura. Nota-se que a produtividade da soja começou a ser penalizada com maior intensidade a partir da segunda quinzena de dezembro e se agravou à medida que a safra avançou no tempo. As três últimas quinzenas analisadas foram as que apresentaram a maior penalização no potencial produtivo, pois a cultura se encontrava nos estádios de florescimento e enchimento de grãos. Nesses estádios, as plantas aumentam a demanda por água e são muito sensíveis ao déficit hídrico que compromete significativamente a produtividade da soja (Sediyama et al., 1996).

O IP acumulado da última quinzena expressa a produtividade obtida pela cultura em relação a sua produtividade potencial máxima, em função da demanda e da disponibilidade meteorológica durante todo o ciclo da cultura. No presente estudo, a maior parte da região considerada indica valores de IP entre 0,4 e 0,5, o que significa uma perda entre 50 e $60 \%$ no potencial produtivo da soja. Isso refletiu-se nos valores de produtividade final estimados pelo modelo AGRO, que foram de 1.797, 1.586 e 1.682 kg.ha ${ }^{-1}$ para os estados do Paraná, São Paulo e Mato Grosso do Sul, respectivamente. Observa-se, ainda, que a porção sul do Mato Grosso do Sul e noroeste do Paraná foram as regiões mais prejudicadas pela estiagem, indicando valores de IP entre 0,1 e 0,2, que refletem uma perda entre 80 e $90 \%$ no potencial produtivo da cultura. Apesar disso, tais regiões concentram poucas lavouras de soja, sobretudo devido à baixa CAD dos solos. 


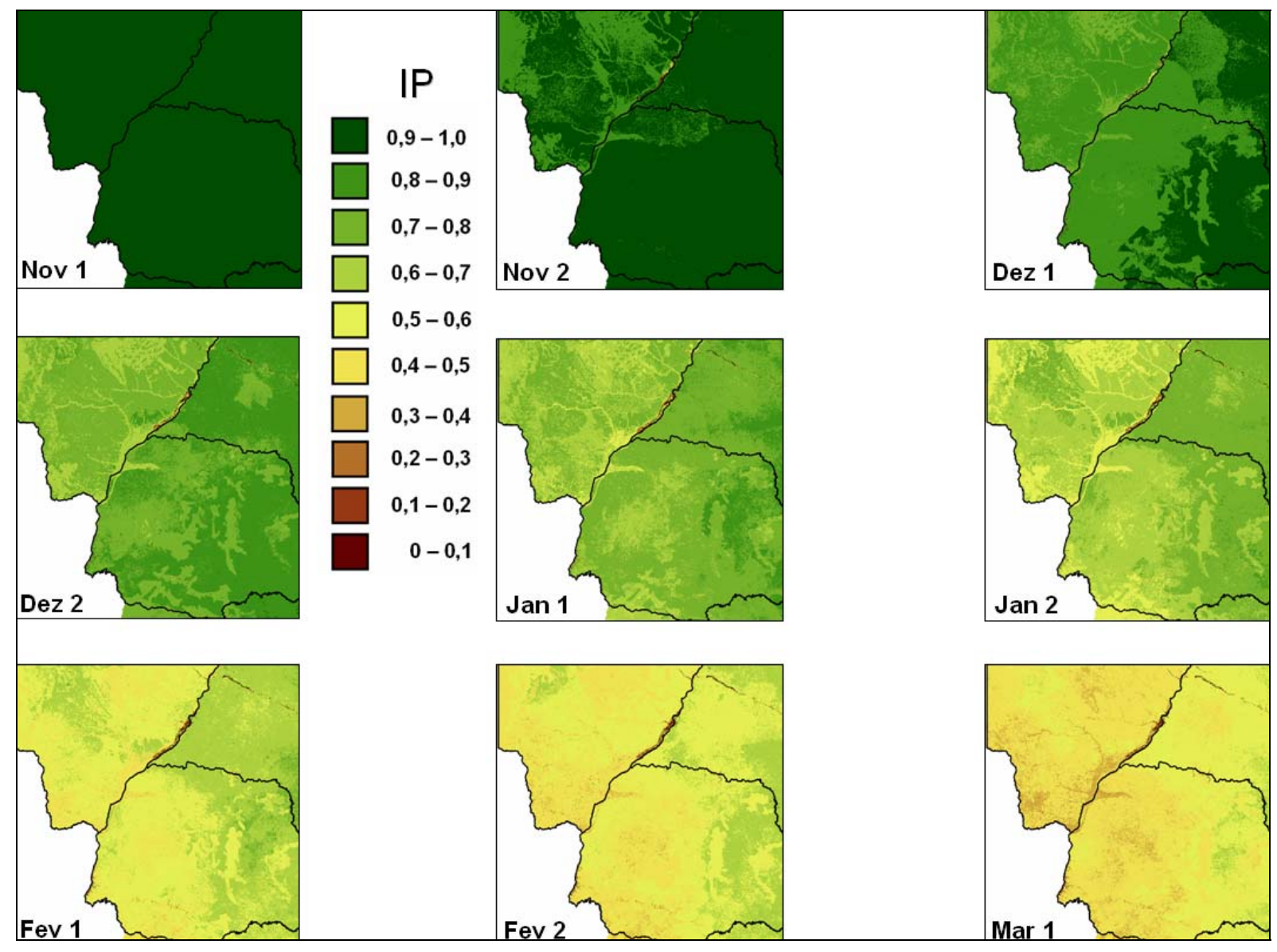

Figura 2. Índices de penalização (IP) quinzenais acumulados para a cultura da soja para a região compreendida pelo estudo, safra 2004/05.

A Figura 3 mostra a produtividade obtida pelo modelo AGRO em comparação à informada pelo IBGE, para os estados de São Paulo, Mato Grosso do Sul e Paraná, para plantio em 01-11-2004. Nota-se que o modelo AGRO, utilizando os dados do modelo Eta, acompanhou a variação interanual dos valores de produtividade para os 3 estados estudados. O Mato Grosso do Sul foi o estado mais atingido pela estiagem, já que tanto o IBGE quanto o modelo AGRO forneceram os menores valores de produtividade. A diferença existente entre a estimativa do IBGE e a do modelo pode estar associada ao fato do IBGE considerar a média estadual que foi consideravelmente superior à produtividade observada na região estudada. Nos três estados houve regiões localizadas fora da área de estudo e que foram menos afetadas pela estiagem, fato que contribuiu para que a média estadual fosse mais elevada.

Enfatiza-se que os dados do modelo Eta podem ser disponibilizados imediatamente após a sua geração, sendo prontamente utilizáveis pelo modelo AGRO, após um simples préprocessamento efetuado por uma rotina computacional. Isso permite a obtenção dos mapas de IP imediatamente após o término da quinzena considerada. É oportuno lembrar, ainda, que o modelo AGRO pode utilizar intervalos de monitoramento menores que o quinzenal (ex. 5, 7 e 10 dias) e ser adaptado para diversas culturas de grande interesse econômico. Além disso, esse modelo pode englobar todas as regiões produtoras do país, já que os dados do Eta são gerados para todo o Brasil e grande parte da América do Sul.

A possibilidade de monitoramento das condições meteorológicas e a avaliação do seu impacto sobre a produtividade potencial das mais variadas culturas, em tempo hábil, são de grande importância para diversos setores do agronegócio, em especial, para o governo. Essas 
informações auxiliam na tomada de decisões, tanto em relação à balança comercial, quanto ao adequado abastecimento interno, auxiliando no estabelecimento de preços justos a produtores e consumidores.

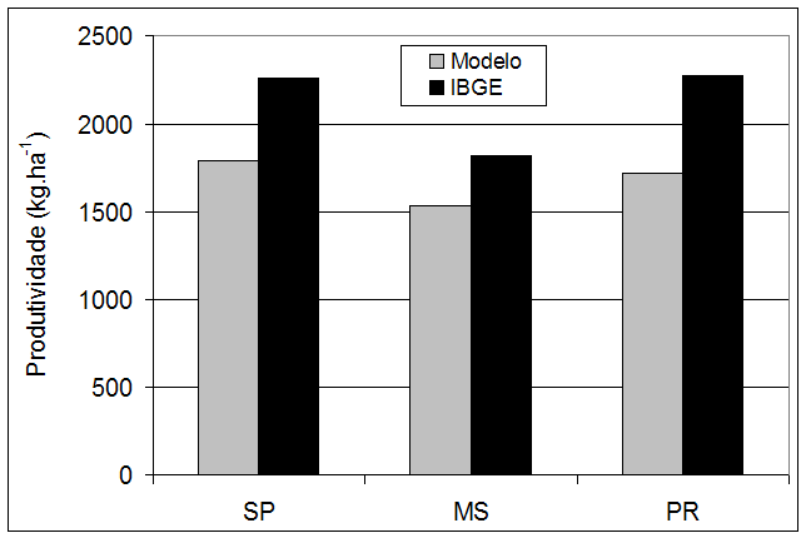

Figura 3. Produtividade obtida pelo modelo AGRO em comparação à informada pelo IBGE, para os estados de São Paulo, Mato Grosso do Sul e Paraná, para plantio em 01-11-2004.

Outra aplicação está relacionada às operações de securidade agrícola. Nesse caso, a identificação dos períodos e das regiões de maior impacto das condições meteorológicas adversas fornece subsídios objetivos para otimizar e tornar mais eficiente o processo de vistoria de campo das lavouras seguradas. Desse modo, pode ser efetuado um direcionamento prioritário às áreas a serem fiscalizadas para avaliação e quantificação das perdas. Esse procedimento impõe um caráter objetivo às vistorias para inibir a possibilidade de fraude e, conseqüentemente, proporcionar um melhor equilíbrio entre o prêmio, pago pelo agricultor, e a indenização, paga pela seguradora.

A despeito da possibilidade da estimativa da produtividade e o monitoramento quinzenal das condições meteorológicas no decorrer da safra de soja em tempo hábil, um fato a ser destacado que se refere aos dados gerados pelo modelo Eta é a regularidade espacial aliada à uniformidade. Em se tratando das estações meteorológicas, além da sua distribuição esparsa e irregular, especialmente nas principais regiões agrícolas, elas são administradas por diferentes instituições. Assim, os dados coletados, em grande parte das vezes, não são disponibilizados de uma maneira integrada, ou seja, no mesmo momento, formato e local, de modo a permitir o monitoramento das culturas, particularmente no que se refere à tomada de decisão em tempo oportuno.

Um local onde são disponibilizados dados coletados por diferentes estações é o portal AGRITEMPO (www.agritempo.gov.br). Mesmo assim, além da baixa densidade das estações em grande parte das regiões agrícolas, apenas estão disponíveis dados referentes à precipitação pluvial e a temperatura (máxima e mínima) do ar, muitas vezes insuficientes para o monitoramento de culturas por meio de modelos agronômicos. Outra opção são os dados coletados automaticamente pelas Plataformas de Coleta de Dados (PCDs) e disponibilizados pelo CPTEC. Todavia, elas incorrem no mesmo problema relacionado à distribuição esparsa e irregular e ao não fornecimento de todos os elementos meteorológicos requeridos por alguns modelos agronômicos. Na Figura 4, visualiza-se a distribuição espacial das PCDs e das estações cujos dados são disponibilizados pelo portal AGRITEMPO, além da grade regular (20 x 20 km) dos dados gerados pelo modelo Eta, para a região compreendida pelo estudo. 


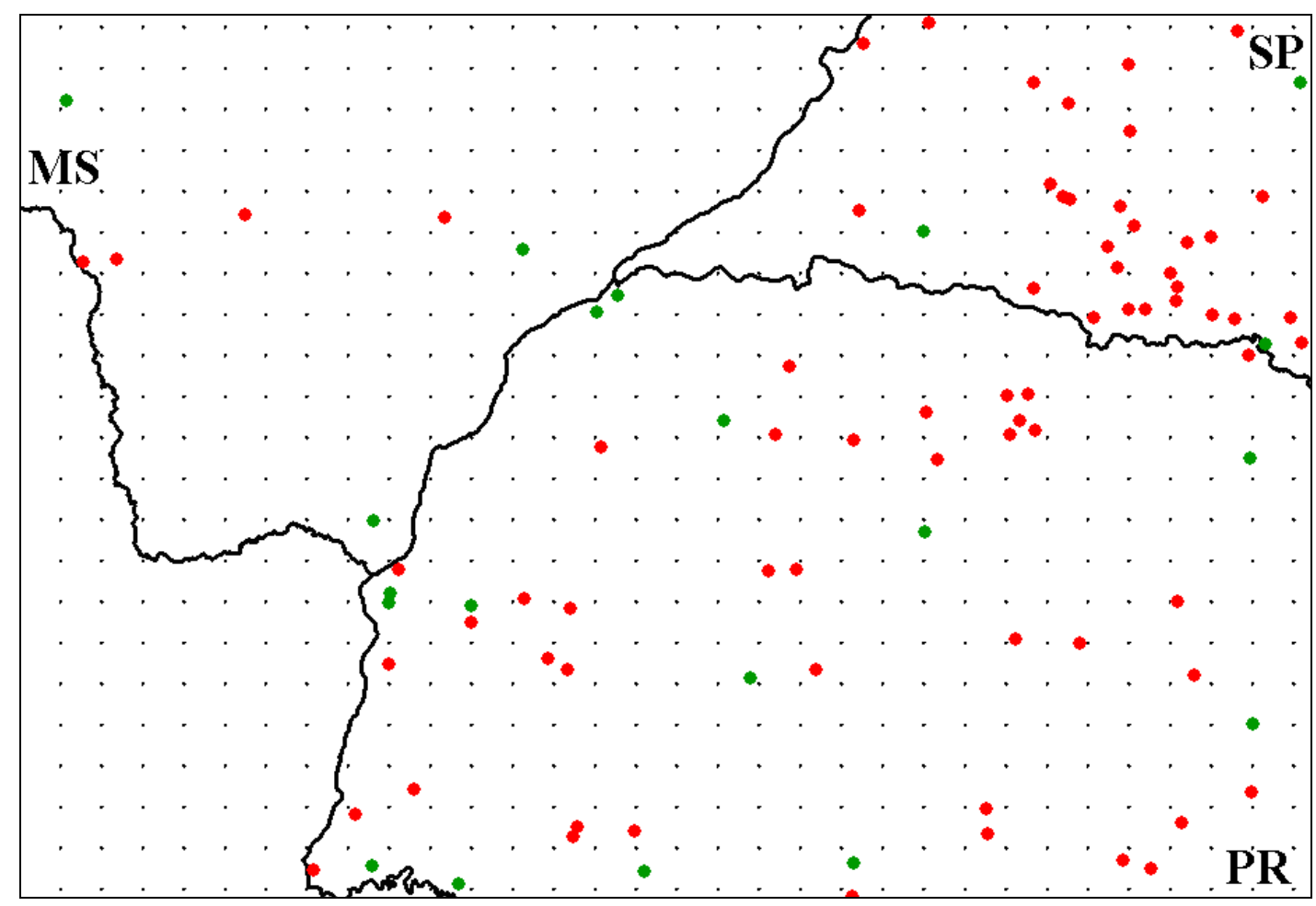

Figura 4. Distribuição espacial das PCDs (esferas verdes), das estações cujos dados são disponibilizados pelo portal AGRITEMPO (esferas vermelhos) e da grade regular (20 x 20 $\mathrm{km}$ ) referente aos dados gerados pelo modelo Eta (pontos pretos), para a região compreendida pelo estudo.

Pela Figura 4 percebe-se claramente a distribuição esparsa e irregular dos pontos amostrais que dizem respeito às PCDs e às estações disponibilizadas pelo AGRITEMPO. Já o modelo Eta fornece um número muito maior de pontos amostrais que estão distribuídos regularmente sobre toda a área considerada no estudo, inclusive além do limite político nacional. Além disso, o caráter operacional da previsão de tempo gerada pelo CPTEC praticamente assegura a regularidade na disponibilização dos dados gerados pelo modelo Eta, reforçando a idéia de que eles podem ser utilizados em métodos objetivos e operacionais de monitoramento de safras e geração de estatísticas agrícolas.

\section{CONCLUSÃO}

Os dados das variáveis meteorológicas gerados pelo modelo regional de previsão de tempo Eta apresentam bom potencial de utilização em modelos agronômicos. Esses dados possibilitam o monitoramento temporal e espacial de culturas agrícolas, além de fornecer a estimativa da produtividade de culturas agrícolas em grandes regiões. O modelo agronômico, em conjunto com os dados do Eta, permite acompanhar o efeito do clima sobre o crescimento e desenvolvimento da cultura da soja, fornecendo informações em tempo hábil para fins de abastecimento do mercado, seguro agrícola entre outros. 


\section{AGRADECIMENTOS}

Os autores expressam agradecimentos à Chou Sin Chan, ao Rildo Gonçalves de Moura e à Andreza Fogaça Coelho do CPTEC por terem auxiliado na disponibilização dos dados meteorológicos gerados pelo modelo Eta.

\section{REFERÊNCIAS}

ALLEN, R. G.; PEREIRA, L. S.; RAES, D.; SMITH, M. Crop evapotranspiration: guidelines for computing crop water requirements. Rome: Food and Agriculture Organization of the United Nations, 1998. 300p. (FAO-Irrigation and Drainage Paper, 56).

BERKA, L. M. S.; RUDORFF, B. F. T.; SHIMABUKURO, Y. E. Soybean yield estimation by an agrometeorological model in a GIS. Scientia Agricola, Piracicaba, v. 60, n. 3, p. 433-440, july/sept., 2003.

BOLTON, D. The computation of equivalent potential temperature. Monthly Weather Review, Boston, v. 108, n. 9, p. 1046-1053, 1980.

BRASIL. Ministério da Agricultura. Departamento Nacional de Pesquisa Agropecuária. Divisão de Pesquisa Pedológica. Levantamento de reconhecimento dos solos do Estado do Rio Grande do Sul. Recife: DNPEA, 1973. 431 p. (Boletim técnico, 30).

CÂMARA, G.; SOUZA, R. C. M.; FREITAS, U. M.; GARRIDO, J. C. P. SPRING: integrating remote sensing and GIS with object-oriented data modelling. Computers \& Graphics, [S.l.], v. 15, n. 6, p. 13-22, july 1996.

CHAN, S. C. Modelo regional Eta. Climanálise, São José dos Campos: CPTEC/INPE, ed. comemorativa, v. 10, out. 1996.

DANG, A.; WANG, X.; WU, H. GIS based study on the potential grain productivity of China. In: INTERNATIONAL GEOSCIENCE AND REMOTE SENSING SYMPOSIUM - IGARSS, 2000, Honolulu. Proceedings... Piscataway: IEEE Publications, 2000. 1 CD-ROM.

DOORENBOS, J.; KASSAM, A. H. Yield response to water. Rome: Food and Agriculture Organization of the United Nations, 1979. 193 p. (FAO-Irrigation and Drainage Paper, 33).

EMPRESA BRASILEIRA DE PESQUISA AGROPECUÁRIA. Sistema brasileiro de classificação de solos. Brasília: Embrapa Produção de Informação, 1999. 412 p.

FARIAS, J. R. B.; NEPOMUCENO, A. F.; NEUMAIER, N.; OYA, T. Ecofisiologia. In: EMPRESA BRASILEIRA DE PESQUISA AGROPECUÁRIA. A cultura da soja no Brasil. Londrina: Embrapa Soja, 2000. 1 CD-ROM.

HARTKAMP, A. D.; WHITE, J. W.; HOOGENBOOM, G. Interfacing geographic information systems with agronomic modellings: a review. Agronomy Journal, Madison, v. 91, n. 5, p. 761-722, sept./oct. 1999. 
HOOGENBOOM, G. Contribution of agrometeorology to the simulation of crop production and its applications. Agricultural and Forest Meteorology, [S.l.], v. 103, n. 1/2, p. 137-157, jun. 2000.

MESINGER, F.; JANJIC, Z. I.; NICKOVIC, S.; GAVRILOV, D.; DEAVEN, D. G. The stepmountain coordinate: model description and performance for cases of alpine lee cyclogenesis and for a case of an Appalachian redevelopment. Monthly Weather Review, Boston, v. 116, n. 7, p. 1493-1518, 1988.

RIZZI, R. Geotecnologias em um sistema de estimativa da produção de soja: estudo de caso no Rio Grande do Sul. 30/04/2004. 214 p. (INPE-12271-TDI/983). Tese (Doutorado em Sensoriamento Remoto) - Instituto Nacional de Pesquisas Espaciais, São José dos Campos. 2004. Disponível em: <http://mtc-m12.sid.inpe.br/rep/sid.inpe.br/ jeferson/2004/07.02.14.17>. Acesso em: 02 jun. 2006. rep: sid.inpe.br/jeferson/ 2004/07.02.14.17.

RUDORFF, B. F. T.; BATISTA, G. T. Yield estimation of sugarcane based on agrometeorological-spectral models. Remote Sensing of Environment, New York, v. 33, n. 3, p. 183-192, 1990.

SEDIYAMA, T.; PEREIRA, M. G.; SEDYIAMA, C. S.; GOMES, J. L. L. Cultura da soja: I parte. Viçosa: Universidade Federal de Viçosa, 1996. 96p. 